[0212-7199 (2008) 25: 3; pp 117-121] ANALES DE MEDICINA INTERNA Copyright (C) 2008 ARAN EDICIONES, S.L.

AN. MED. INTERNA (Madrid) Vol. 25, N. ${ }^{\circ} 3$, pp. 117-121, 2008

\title{
Neumonía en el anciano mayor de 80 años con ingreso hospitalario
}

\author{
G. ZUBILLAGA GARMENDIA, E. SÁNCHEZ HAYA, J. BENAVENTE CLAVERAS, \\ E. CECIAGA ELEXPURU, I. ZAMARREÑO GÓMEZ, E. ZUBILLAGA AZPÍROZ, \\ C. SARASQUETA EIZAGUIRRE'
}

Servicios de Medicina Interna y ${ }^{\prime}$ Epidemiología. Hospital Donostia. Servicio Vasco de Salud. Departamento de Medicina. Universidad del País Vasco (UPV-EHU)

PNEUMONIA ABOVE 80 YEARS, ADMITTED TO THE HOSPITAL

\section{RESUMEN}

Objetivo: analizar y comparar diferencias en pacientes mayores de 80 años con Neumonía Adquirida en la Comunidad (NAC) como diagnóstico de alta en pacientes ingresados desde Urgencias a servicios de Medicina Interna (MEDIN) y Neumología (NEUMO) de un Hospital General.

Material y métodos: análisis retrospectivo de todos los 277 pacientes mayores de 80 años con ingreso hospitalario por NAC en el 2005.

Resultados: $84 \%$ Comunitarios. $16 \%$ de Instituciones. Edad media: 85,8 años (52\% mujeres). Severidad: 19\% FINE-3. 49\% FINE-4. 32\% FINE-5. Etiología conocida 25\% (Neumocócica 19\%, H. Influenzae y otros Gram (-) 6\%). 75\% trata MEDIN, 22\% NEUMO. Guías Clínicas de tratamiento estrictas 30'5\%, una variante 60\% (Igual en MEDIN que en

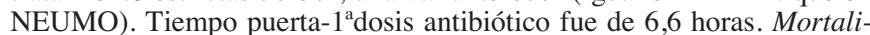
dad: $16,7 \%$. Edad fallecidos mujeres: 87,4 a. mayor que hombres: 84,5 a. $(\mathrm{p}=0,035)$. Mortalidad FINE-3-4-5: 4,5, 12,4,30\% respectivamente. Mortalidad tratados antes de 4 horas: $34,6 \%$, después de 4 horas: 11,5\% $(\mathrm{p}=0,01)$. Mucho más FINE-5 en MEDIN que en NEUMO. Mortalidad MEDIN: $22 \%$, Mortalidad NEUMO: $3 \%(\mathrm{p}=0,001)$. Mortalidad igual siguiendo la Guía Clínica o variante.

Conclusiones: a) MEDIN recibe pacientes más graves que NEUMO; b) mortalidad importante $(16,7 \%)$ y progresiva en la escala FINE, a pesar de tratamiento correcto; c) la rápida administración de antibióticos no redujo la mortalidad; d) la mortalidad no varía aún con variantes de las Guías Clínicas; y c) hay areas de mejora en nuestros Servicios.

\section{ABSTRACT}

Objetive: To analize and compare differences in patients older than 80 years with Community acquired Pneumonia admitted in Internal Medicine or Pneumology of a General Hospital from the Emergency Room.

Material and methods: Retrospective study of all the 277 patients above 80 years admitted into the Hospital in 2005 with the main diagnosis of Pneumonia.

Results: $84 \%$ community-acquired, $16 \%$ from Institutions. Mean age: 85.8 y (48\% men, $52 \%$ women). $19 \%$ FINE-3, $49 \%$ FINE-4, $32 \%$ FINE-5. Known etiology: 25\% (Pneumococcal 19\%, H. Influenzae and other Gram (-) 6\%. 75\% treated by Internists, $22 \%$ treated by Pneumologists. Standard Guidelines followed up by 30,5\% a variant $60 \%$ (Equal by Internists or Pneumologists). Time door-1st antibiotic dose 6.6 hours. Global Mortality 16.7\%. Women died at 87.4 y, men at 84.5 y $(p=$ 0.035). Mortality FINE 3-4-5: 4.5, 12.4, 30\% respectively. Mortality treated before 4 hours: $34.6 \%$, after 4 hours: $11.5 \%(p=0.01)$. Many more FINE 5 cases in Int. Medicine than Pneumology. Mortality by Internists 22\%. Mortality by Pneumologists 3\% $(p=0.001)$. Mortality similar following strict guidelines or variant.

Conclusions: a) Internist receive patients sicker than Pneumologists; b) Important mortality in these very old patients of $16.7 \%$, and progressive according the FINE severiy index, in spite of correct therapy; c) Rapid initiation of Antibiotics did not decreased mortality; d) Mortality did not change following strict or variant Guidelines; and e) There are areas of quality improvement in our Hospitals.
PALABRAS CLAVE: Neumonía. Anciano. Tiempo puerta-antibiotico. Medicina Interna. Neumología.
KEY WORDS: Pneumonia. Elderly. Aged, 80 and over. Treatment. Therapy. Hospitalist.

Zubillaga Garmendia G, Sánchez Haya E, Benavente Claveras J, Ceciaga Elexpuru E, Zamarreño Gómez I, Zubillaga Azpíroz E, Sarasqueta Eizaguirre C. Neumonía en el anciano mayor de 80 años con ingreso hospitalario. An Med Interna (Madrid) 2008; 25: 117-121.

\section{INTRODUCCIÓN}

Las neumonías son la primera causa de muerte de enfermedad infecciosa en el anciano. La mortalidad con tratamiento varía desde el $4 \%$ a más del $60 \%$ según el origen domicilio o residencias, las comorbilidades, edades, gérmenes causales, prontitud en la instauración del tratamiento, ventilación mecánica, antibiótico(s) utilizados, e intensidad de control de los mismos. La mortalidad según las edades varía, siendo elevada en los muy mayores. La escala de FINE (1) (PSI -Pneumonia severity index) es una nueva medida de juzgar la gravedad de las mismas. Nuevas recomendaciones y guías $(2,11)$ hablan de la instauración de diferentes tratamientos en función de la gravedad, y el mantenimiento de los antibióticos durante un tiempo suficiente (IV inicial, luego oral en muchos casos), estas guías se han realizado por estudios prospectivos; pero en los

Trabajo aceptado: 19 de noviembre de 2007 
estudios existen pocos ancianos (3). Ancianos de 90 años con neumonía y ventilación mecánica tienen mortalidades de hasta el $55 \%$. Estas guías clínicas se aplican a ancianos de la cuarta edad (mayores de 80 años). Como objetivo queremos: a) conocer el número de pacientes con Neumonía mayores de 80 años tratados en nuestro Hospital Donostia durante el año 2005, características demográficas de los mismos; b) gravedad en la escala de FINE de las mismas; c) identificar factores de riesgo de mortalidad: medir su asociación con edad, sexo, origen de comunidad o institución, FINE, servicio, antibióticos utilizados de inicio. Mortalidad de estos pacientes según sean de comunidad o residencias, según etiologías; d) cumplimiento de las guías de práctica clínica en el tratamiento de las mismas (antibióticos utilizados, tiempo de inicio de antibióticos); y e) servicios que tratan estos pacientes, diferencias en los resultados.

\section{MATERIAL Y MÉTODO}

Estudio retrospectivo en el que obtenemos del CMBD (conjunto mínimo básico de datos) de documentación clínica del hospital todas las 277 historias con diagnóstico de alta de neumonía de comunidad mayores de 80 años del año 2005 concordantes en clínica y radiología. La variable dependiente estudiada fue la mortalidad al alta. Las variables independientes analizadas en el presente estudio han sido edad, sexo, comorbilidades según la escala de FINE, y grado de severidad correspondiente, lugar de origen (domicilio o institución). destino al alta (domicilio, institución, fallecidos, hospital a domicilio, traslado a centro de crónicos). Obtenemos el tratamiento iniciado, tiempo de instauración de la primera dosis de antibióticos desde la llegada a Urgencias. Agentes causales de la enfermedad en los que se pueda determinar, si bien, en general, el tratamiento se inicia empíricamente y se sigue así habitualmente. Servicios que tratan, comparación de factores de riesgo entre servicios, comparación de mortalidad entre servicios. Análisis estadístico: se han utilizado proporciones y medias para describir las variables cualitativas y cuantitativas respectivamente. Para medir la asociación de variables cualitativas con la mortalidad se ha aplicado el test de Fisher o el test de Chi-cuadrado y en el caso de las cuantitativas el test de student. Finalmente se ha ajustado un modelo de regresión logística binaria para determinar las variables que independientemente de posibles factores de confusión se asocien con la probabilidad de éxitus.

\section{RESULTADOS}

En la tabla I describimos la muestra. De la etiología de Gram(-)vos: hubo dos aislamientos de E. coli, 2 Enterococ. Cloacae, 2 Stenot. Maltophilia, 1 SAMR, 1 Staph Coagul(-)vo. No atípicas. La edad media de ingresos era similar en ambos servicios $(p=0,67)$.

Ingresan en MEDIN doble, triple y cinco veces más pacientes FINE 3, 4 y 5 que en NEUMO en número absoluto. En la tabla II exponemos las variables asociadas a mortalidad como sexo, edad, origen, FINE, etiología, tiempo de inicio antib., servicio, seguimiento de guías clínicas. En la tabla III exponemos los resultados del riesgo relativo (odds ratio) según el análisis multivariante.

\section{TABLA I}

\section{DESCRIPCIÓN DE MUESTRA}

\author{
Edad: 85,8 (DS 4,5) a. \\ Rango edad: 80-99 a. \\ Sexo: $52 \%$ mujeres \\ Origen: \\ Domicilio: $84 \%$ \\ Institución: $16 \%$ \\ FINE (PSI): realizado a 270 pacientes \\ FINE1 $=\mathrm{N}^{\circ}$ pacientes \\ FINE2 $=2(0,7 \%)$ \\ FINE3 $=51(18,8 \%)$ \\ FINE4 $=131(48,5 \%)$ \\ FINE5 $=86(32 \%)$ \\ Etiologías: \\ Desconocida: $75 \%$ \\ Neumoc.: 20\%. H. Influe.: 2,2\% \\ Otros Gram (-)vos: $3 \%$. \\ Tratamiento: \\ B-lactam.+Macról.o Quinol. 30,5\% \\ Beta-lactam. \\ Carbapen \pm Aminoglic. $60 \%$ \\ No consta \\ Fallecen antes de inicio 10\% \\ Tiempo puerta-1 ${ }^{\text {er }}$ Antib.: 6,6 horas \\ Servicios: \\ M. Interna $75,5 \%$ \\ Neumología 22,7\% \\ Otros 1,8\% \\ Edad media similar: \\ Medin 85,5 a. \\ Neumo 85,3 a. \\ Destino Alta: \\ Domicilio 67\% \\ Fallecimiento $16,7 \%$
}

\section{DISCUSIÓN}

Analizamos en nuestro estudio retrospectivo todas las NAC (277) del año 2005 mayores de 80 años en la práctica general hospitalaria. Observamos una casi igualdad de hombres (48\%) y mujeres (52\%). La edad media de 85,8 años refleja la gran mayoría entre 80 y 90 años, unos pocos mayores de 90 años, alguno llegando a los 99 años.

No hay diferencia entre la edad media de los hombres (85,1 años) y mujeres (86,5 años). La procedencia de los pacientes de domicilio 84 y 16\% de una Institución reflejan la demografía de nuestra comunidad. Pacientes con elevados grados de FINE-3,4,5 reflejan ingresos apropiados, comorbilidades importantes y riesgo grave o muy grave. El desconocimiento de la etiología es común en la mayoría de NAC, siendo lo habitual alrededor del 70\%; en nuestra serie de ancianos es del $75 \%$, a pocos pacientes se les realizan todas las pruebas diagnósticas etiológicas, pocas veces tienen esputo, y siempre se inicia un tratamiento empírico que se puede modificar después según resultados.

La mortalidad global del $16,7 \%$ en nuestros pacientes es similar al estudio prospectivo de Zalacain y cols. (4) cuya mortalidad para ancianos mayores de 80 años fue del 17,3\% (25 de 


\section{TABLA II}

VARIABLES ASOCIADAS A MORTALIDAD

Sexo: no diferencia significativa

Valor de $p$

Edad Fallec.:

Hombres: 84,5 a.

Mujeres: 87,4 a.

Origen:

Domicilio: $15 \%$

Institución: 25,6\%

FINE (PSI)- mortalidad global: 16,7\%

Según FINE 2: 0\%

Según FINE 3: 4,5\%

Según FINE 4: 12,4\%

Según FINE 5: 30,1\%

Mortalidad-etiología:

Desconocida $42,5 \%$

Neumococo $7,8 \%$

H. Influenzae $16,7 \%$

Gram (-)vos 33\%

Mortalidad tiempo puerta- $1^{\text {er }}$. antibiótico

Menor de 4 horas: $34,6 \%$

Mayor de 4 horas: $11,5 \%$

Servicio fallecimiento:

Medin: $20,2 \%$

NEUMO: $3,2 \%$

Seguimiento guía clínica:

Standard $14,2 \%$

No estricto $17,6 \%$

No diferencias seguimiento guías

Clínicas Medin o Neumo.

TABLA III

VARIABLES QUE INDEPENDIENTEMENTE DE FACTORES DE CONFUSIÓN SE ASOCIAN A MORTALIDAD (ANÁLISIS

MULTIVARIANTE DE REGRESIÓN LOGÍSTICA BINARIA)

Sexo: odds ratio: 2,2 mujeres vs. hombres.

FINE 4 vs FINE 3: odds ratio 3,2.

FINE 5 vs FINE 3: odds ratio 9,2.

Tiempo inicio tratamiento: menor de 4 horas.

Servicio MEDIN vs. NEUMO: 5,2.

144 pacientes), y similar al 16,2\% en el estudio de Saldías y cols. (5), para mayores de 83 años. En el estudio de Kaplan y cols. (3), la mortalidad fue del 10 al $23 \%$ en ausencia o presencia de disfunción orgánica. La mortalidad era mayor en los $H$. Influenzae y otros gram negativos que en la neumocócica.

También es habitual que la mortalidad sea superior en los provenientes de instituciones o residencias, 25,6\% en nuestro estudio, similar al 28\% de Martínez-Moragón (6) y cols. y del $43 \%$ en el Zalacain y cols., y $17,6 \%$ en el estudio de Kaplan.

La mortalidad según los grados de FINE es acorde (1) con la literatura (4,5\% en el Grupo FINE 3), (12,4\% en el Grupo FINE 4), (30\% en el Grupo FINE 5).

El destino al alta refleja escaso uso del hospital a domicilio, solamente $1,1 \%$.

La mortalidad puede incrementarse en el mes siguiente de seguimiento post-hospitalario (5) como se ha documentado hasta en un $3 \%$.
Según las guías clínicas, empíricamente el tratamiento con betalactámicos (amoxicilina con o sin clavulánico) más macrólidos debiera ser la pauta habitual o las nuevas quinolonas (levofloxacino o moxifloxacino), o betalactámicos más quinolonas; pero en nuestra revisión cualquiera de las combinaciones anteriores lo realizan sólo un 30'5\%, siendo de hecho mayor en MEDIN, sin diferencia significativa. Se prescribe más frecuentemente $(60 \%)$ sólo betalactámicos o añadidos a aminoglicósidos o carbepenémicos, por la sospecha de tratarse de gram negativos que confieren mayor riesgo a la neumonía y la baja relevancia de neumonías por gérmenes atípicos, incluida legionella en estas edades. Algunas neumonías son por otros gram negativos (Pseudomonas, E. coli, etc.), $4 \%$ en nuestro estudio, pero la gran mayoría son de origen indeterminado, por lo que sería recomendable seguir las guías, no obstante, y modificar a posteriori la pauta de antibiótico, si se confirmara otro germen. En nuestro estudio a 84 pacientes que se les aplicó la guía clínica, murieron 12 (14,2\%) y a 193 pacientes que no se les aplicó estrictamente la guía clínica murieron 34 pacientes $(17,6 \%)$, esa diferencia no es significativa $(\mathrm{p}=0,3)$. Por tanto, no podemos acusar el no cumplimiento de la guía clínica como causa de mortalidad. En otros estudios $(2,7,8)$, no hay una unanimidad de uso preferente. De hecho un metaanálisis (10) de 24 trabajos con 5.015 pacientes no encontraba argumentos de mejoría clínica, mortalidad, ni efectos adversos para justificar la cobertura de gérmenes atípicas en las neumonías de comunidad que ingresan en hospitales en general, salvo cuando existían infecciones por legionella.

La administración de la primera dosis de antibióticos en nuestro hospital está en 6,6 horas. La administración de la primera dosis de antibiótico se debiera realizar en Urgencias lo antes posible $(8,13,14)$, una vez realizada la historia clínica, exploración, radiología de tórax, muestras analíticas, toma de hemocultivos y cultivos pertinentes (esputo, orina, etc.), y antes de ser enviado a planta médica que demora el tratamiento. Esta práctica se ha considerado adecuada como de 8 horas en el tiempo puerta-primera dosis de antibiótico y 24 horas en el hemocultivo $(9,26)$. Algunos pacientes han tomado antibióticos antes de llegar al hospital. Eso reduce la mortalidad. El tiempo de 8 horas se ha considerado excesivo en otro estudio (14) retrospectivo extenso de 13.771 pacientes sin antibiótico previo, no exclusivo de muy ancianos, que recibieron el antibiótico en las primeras 4 horas de llegar a Urgencias, descendiendo la mortalidad hospitalaria de 7,4 a $6,8 \%$ y a los 30 días de 12,7 a $11,6 \%$ si recibían el antibiótico las primeras 4 horas, y reduciendo la estancia hospitalaria media en 0,4 días. Otro estudio prospectivo (15) con 409 pacientes, en los que la mitad habían recibido antibióticos en las primeras 4 horas y la otra mitad después, no encontró diferencias en el tiempo hasta la mejoría clínica, ni diferencia en mortalidad; se vio que lo que marcaba el retraso del inicio de antibióticos era la confusión mental, la ausencia de fiebre e hipoxia inicial, y la edad avanzada. En el estudio de Metersky y cols. (17), hasta el $22 \%$ de pacientes no tenían en las primeras 4 horas criterios de neumonía en base a ausencia de estertores, infiltrado y pulsioximetría normal. La administración inmediata de antibiótico llevaría a abuso de los mismos y resistencias bacterianas (19). Hasta el momento, no existe evidencia en cuanto a la mortalidad a los 30 días para justificar esa necesidad de acelerar la administración de antibióticos de 8 a 4 horas $(9,13)$ a todos los pacientes. Una rapidez excesiva de administrar anti- 
bióticos conllevaría a tratar procesos clínicos probables de neumonía sin evidencia de la misma. La mejoría del proceso clínico no está solamente en ese tiempo de primera dosis, sino en los riesgos de base y los cuidados posteriores que influyen decisivamente $(12,16)$.

En nuestro estudio se administran antibióticos, en 6,6 horas desde la llegada a puerta; que está entre la 8 y las 4 horas de la referencias descritas. Si comparamos la mortalidad en la administración del antibiótico antes de las 4 horas o después, obtenemos que la mortalidad en la administración precoz es de $34,6 \%$ y en los de más de 4 horas $11,5 \%(p=0,01)$, resultado inverso al esperado. En el subgrupo de FINE 4 no había diferencia entre menos de 4 horas y más de 4 horas. Sin embargo, en los FINE 5 (muy severos) la mortalidad era del $66 \%$ administrando el antibiótico antes de las 4 horas y $21 \%$ si se administraba después de 4 horas, lo que refleja que a los muy graves en Urgencias les aplican el antibiótico y en cambio, a los no tan graves o con diagnóstico más dudoso, se espera más tiempo; pero por el hecho de menor gravedad tienen menos mortalidad de base, independiente del antibiótico. No siempre se administra el antibiótico en Urgencias, muchas veces se prescribe la pauta en las órdenes de Urgencias o Planta y enfermería proporciona la primera dosis cuando suministra farmacia o en el turno siguiente, en el horario habitual de medicación. Habría que insistir en administrar los antibióticos antes de 4 horas desde la llegada como recomiendan las guías y preferentemente en Urgencias la primera dosis, sobre todo en pacientes mayores de 65 años, como los nuestros, que tengan evidencia radiológica y no hubieran recibido antibiótico previo ambulatorio (18). Este es un area de mejora del tratamiento de las neumonías.

Los servicios que tratan estos pacientes son los habituales de Medicina Interna y Neumología. En el análisis comparativo que realizamos de los pacientes que ingresan en MEDIN y NEUMO observamos similar edad al ingreso, mayores comorbilidades en MEDIN independiente del FINE. En cuanto al seguimiento de las guías de tratamiento, es similar el uso de betalactámicos y macrólidos o quinolonas en ambos servi- cios, por lo que la diferencia en la mortalidad no se debe a un peor tratamiento de los pacientes, sino en la selección inicial desde Urgencias a un servicio u otro, en función de la aparente mayor o menor comorbilidad, y algún factor de terminalidad no incluido en el FINE. En este sentido, Capelastegui y cols. (20) en un estudio sí encuentran diferencias en la cobertura de gérmenes atípicos, menor estancia hospitalaria, menor mortalidad al alta y a los 30 días, en NEUMO que en MEDIN, pero también en su estudio los enfermos de MEDIN eran mayores, provenientes de residencias, con mayores comorbilidades, con diferencias muy significativas, y la administración del antibiótico era similar en ambos servicios antes de 8 horas. El empleo de macrólidos en nuestro estudio ha sido similar en MEDIN que en NEUMO, aunque no tenga tanta justificación como en pacientes de menor edad, dado que no hay gérmenes atípicos en estas edades. En cuanto al perfil del servicio para tratar idóneamente estos pacientes, el estudio de Capelastegui refuerza el compromiso de conocimiento, actualización y experiencia para iniciar una rápida instauración del tratamiento, bien protocolizado, paso de medicación IV a oral, estancias hospitalarias óptimas y un buen seguimiento aplicables a todos los servicios (21). Diferentes modelos de especialistas y generalistas, más por interés de trabajo profesional, para tratar estos pacientes se han investigado en otros paises (22-25) y no parece haber diferencias en los resultados, si bien se inclinan por el modelo de médico hospitalista con dedicación y experiencia, como ocurre en nuestros hospitales; siendo importante encontrar esos nichos de mejora y aplicarlos en el futuro.

En conclusión; la NAC del anciano mayor de 80 años tiene una mortalidad importante $16,7 \%$, aún con buen tratamiento, aplicado en 6,6 horas tras recepción en Urgencias. Edad mayor en ancianas fallecidas. La mortalidad asciende al $30 \%$ en los FINE-5. MEDIN recibe enfermos más graves. NEUMO tiene menor mortalidad por algún factor pronóstico no recogido en el FINE. Se debieran realizar estudios prospectivos comparativos con aplicación del antibiótico antes de 4 horas y ver si se reduce la mortalidad, retrospectivos como éste, no lo demuestran. Hay posibilidad de mejora de calidad en estos procesos.

\section{Bibliografía}

1. Fine MJ, Auble TE, Yelay DM, et al. A prediction rule to identify lowrisk patients with Community-Acquired Pneumonia. N Engl J Med 1997; 336: 243-50.

2. American Thoracic Society. Guidelines for the Management of Adults with Community-Acquired Pneumonia. Am J Respir Crit Care Med 2001; 163: 1730-54.

3. Kaplan V, Angus D, Griffin M, Clermont G, Watson S, Linde-Zwirble W. Hospitalized Community-Acquired Pneumonia in the elderly. Am J Respir Crit Care Med 2002; 165: 766-72.

4. Zalacain R, Torres A, Celis R, Blanquer J, Aspa J, Esteban et al. Community-Acquired Pneumonia in the elderly. Spanish multicentre study. Eur Respir J 2003; 21: 294-302.

5. Saldías F, O’Brien A, Gederlini A, Farías G, Díaz A. Neumonía Adquirida en la Comunidad en el anciano inmunocompetente que requiere hospitalización. Cuadro clínico, factores pronósticos y tratamiento. Arch Bronconeumol 2003; 39: 333-40.

6. Martínez-Moragón E, García L, Serra B, Fernández E, Gómez A, Jukve R. La Neumonía Adquirida en la Comunidad de los ancianos: diferencias entre los que viven en Residencias y en domicilios particulares. Arch Bronconeumol 2004; 40: 547-52.

7. Gordon GS, Throop D, Berberian L, Niederman M, Bass J, Alemayehu $\mathrm{D}$, Mellis S. Validation of the therapeutic recommendations of the Ame- rican Thoracic Society (ATS) guidelines for Community-Acquired Pneumonia in hospitalized patients. Chest 1996; 110: 55S.

8. Gleason PP, Meehan TP, Fine JM, Galusha DH, Fine MJ. Associations between initial antimicrobial therapy and medical outcomes for hospitalized elderly patients with Pneumonia. Arch Intern Med 1999; 159: 2562-72.

9. Meehan TP, Fine MJ, Krumholz HM, Scinto JD, Galusha DH, Mockalis JT, et al. Quality of care, process, and outcomes in elderly patients with Pneumonia. JAMA 1997; 278: 2080-4.

10. Shefet D, Robenshtok E, Paul M, Leibovici L. Empirical Atypical coverage for Inpatients with Community-Acquired Pneumonia. Arch Intern Med 2005; 165: 1992-2000.

11. Alfageme J, Aspa J, Bello S, Blanquer R, Borderías L et al. Normativa para el diagnóstico y tratamiento de la Neumonía Adquirida en la Comunidad. Sociedad Española de Neumología y Cirugía Torácica (SEPAR). Arch Bronconeumol. 2005; 41: 272-89.

12. Menéndez R, Torres A, Rodriguez de Castro F, Zalacain R, Aspa J, Martín JJ, et al. Reaching stability in Community-Acquired Pneumonia: The effects of the severity of disease, treatment, and the characteristics of patients. Clin Infect Dis 2004; 39: 1783-90.

13. Battleman DS, Callahan M,Thaler HT. Rapid antibiotic delivery and appropiate antibiotic selection reduce length of Hospital stay of patients 
with Community-Acquired Pneumonia. Arch Intern Med 2002; 162: 682-8.

14. Houck PT, Bratzler DW, Nsa W, Ma A, Bartlett JG. Timing of antibiotic administration and outcomes for Medicare patients hospitalized with Community-Acquired Pneumonia. Arch Intern Med 2004; 164: 637-44.

15. Silber SH, Garrett C, Singh R, Sweeney A, Rosenberg C, Parachiv D, Okafo T. Early administration of antibiotics does not shorten time to clinical stability in patients with moderate-to-severe Community-Acquired Pneumonia. Chest 2003; 124: 1798-804.

16. Waterer GW, Kessler LA, Wunderink RG. Delayed administration of antibiotics and atypical presentation in Community-Acquired Pneumonia. Chest 2006; 130: 11-5.

17. Metersky ML, Sweeney TA, Getzow MB, Siddiqui F, Nsa W, Bratzler DW. Antibiotic timig and diagnostic uncertainty in Medicare Patients with Pneumonia. Is it reasonable to expect all patients to receive antibiotics within 4 hours? Chest 2006; 130: 16-21.

18. Houck PM. Antibiotics and Pneumonia: Is timing everything or just a cause of more problems? Chest 2006; 130: 1-3.

19. Kanwar M, Brar N, Khatib R, Fakih M. Misdiagnosis of CommunityAcquired Pneumonia and inappropiate utilization of Antibiotics. Chest 2007; 131: 1865-9.

20. Capelastegui A, España PP, Quintana JM, Gorordo I, Martínez Urquiri
A, Idoiaga I, Bilbao A. Paciente ingresados por Neumonía Adquirida en la Comunidad: estudio comparativo en función de la especialidad del servicio médico responsable. Arch Bronconeumol 2005; 41: 300-6.

21. Rodríguez de Castro F. Influencia de la especialidad en el manejo de la Neumonía hospitalizada. Arch Bronconeumol 2005; 41: 297-9.

22. Dean NC, Silver MP, Bateman KA. Frequency of subspecialty physician care for elderly patients with Community-Acquired Pneumonia. Chest 2000; 117: 393-7.

23. Meehan TP, Chua-Reyes JM, Tate J, Prestwood KM, Scinto JD, Petrillo MK, Metersky ML. Process of care performance, patient characteristics, and outcomes in elderly patients hospitalized with Community-Acquired or nursing home-acquired Pneumonia. Chest 2000; 117: 1378-85.

24. Smith PC, Westfall JM, Nichols RA. Primary care family physicians and 2 hospitalist models: comparison of outcomes, processes, and costs. J Fam Pract 2002; 51: 1021-7.

25. Rifkin WD, Conner D, Silver A, Eichrorn A. Comparison of processes and outcomes of Pneumonia care between hospitalist and communitybased primary care physicians. Mayo Clin Proc 2002; 77: 1053-8.

26. Fine JM, Fine MJ, Galusha D, Petrillo M, Meehan MD. Patient and Hospital Characteristics associated with recommended processes of care for elderly patients hospitalized with Pneumonia. Arch Intern Med 2002; 162: 827-33. 